\title{
Radial Head Resection versus Arthroplasty in Unrepairable Comminuted Fractures Mason Type III and Type IV: A Systematic Review
}

\author{
Francesco Catellani (D), Francesca De Caro, Carlo F. De Biase, Vincenzo R. Perrino, \\ Luca Usai, Vito Triolo, Giovanni Ziveri, and Gennaro Fiorentino \\ Department of Orthopaedics and Traumatology, Istituto Clinico Humanitas Gavazzeni, Bergamo 24125, Italy \\ Correspondence should be addressed to Francesco Catellani; francesco.catellani@gavazzeni.it
}

Received 11 March 2018; Accepted 3 July 2018; Published 16 July 2018

Academic Editor: Peter Angele

Copyright (C) 2018 Francesco Catellani et al. This is an open access article distributed under the Creative Commons Attribution License, which permits unrestricted use, distribution, and reproduction in any medium, provided the original work is properly cited.

Unrepairable comminuted fractures of the radial head Mason type III or type IV have poor outcomes when treated by open reduction and internal fixation. Radial head resection has been proposed as good option for surgical treatment, while in the last decades, the development of technology and design in radial head prosthesis has increased efficacy in prosthetic replacement. The present review was conducted to determine the best surgical treatment for comminuted radial head when ORIF is not possible. Better outcomes are reported for radial head arthroplasty in terms of elbow stability, range of motion, pain, and fewer complications compared to radial head excision. Nevertheless, radial head resection still can be considered an option of treatment in isolated radial head fractures with no associated ligament injuries lesion of ligaments or in case of older patients with low demanding function.

\section{Introduction}

Surgical treatment for comminuted and unrepairable fractures of the radial head may be challenging. These types of fractures are often associated with multiple ligamentous injuries amounting to elbow instability. Radial head resection has been proposed as good option for surgical treatment, while in the last decades, the development of technology and design in radial head prosthesis has increased efficacy in prosthetic replacement.

The radial head is a secondary valgus stabilizer of the joint and it is involved in transmission of axial force load through the elbow during flexion [1]. It is also a varus and external rotatory constrainer [2]. Comminuted radial head fractures Mason type III and type IV are commonly associated with other injures of the elbow as capitellum and coronoid fractures and/or ligaments disruption, both medial and lateral ligaments and interosseus membrane [3-6]. Primary goal in surgical treatment is to restore elbow stability in order to preserve the complex physiologic elbow kinematics. In this respect, medial collateral ligament is the primary constrainer in valgus stress. Radial head contributes secondarily to valgus stability $[1,7]$ and its preservation is mandatory in case of fractures that involve soft tissue and ligaments to avoid chronic instability. Many authors have described serious complications in case of resection of the radial head such as proximal migration of radius and longitudinal instability, humeroulnar osteoarthritis [2, 7-9], decrease in grip strength, cubitus valgus, and ulnar neuropathy $[10,11]$. Therefore, radial head arthroplasty has obtained a large consensus in orthopaedic surgeons as primary option of treatment in fractures Mason types III and IV. It allows an anatomical reconstruction and it maintains stability and physiologic kinematics of the elbow if associated with ligament reconstruction. However, oversizing or overstuffing of radial head prosthesis, malpositioning, and aseptic mobilization may lead to a high rate of complications and failure of this surgical procedure. Recent reviews of literature $[10,12]$ on elbow arthroplasties have reported satisfactory results in radial head replacement studies due to improvement of biomaterials and operative techniques.

The purpose of this review was to investigate the current literature on surgical treatment of unrepairable comminuted 


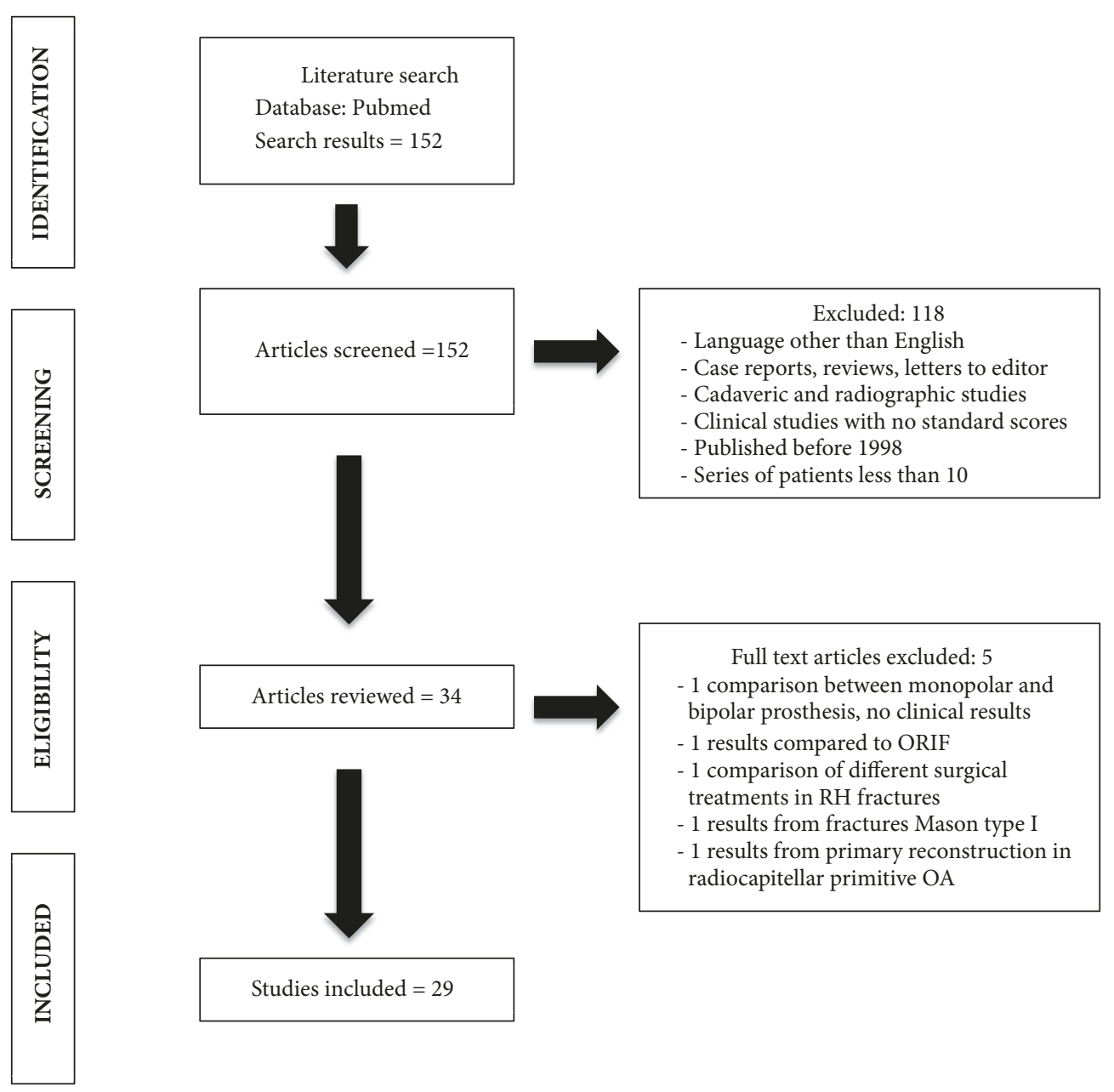

Figure 1

radial head fractures Mason type III or type IV to assess results and indications for radial head replacement or resection.

\section{Materials and Methods}

We searched in PubMed electronic database the words (radial head fractures) AND ((artrhoplasty) OR (prosthesis)) AND ((resection) OR (excision)). The guidelines for preferred reporting items for systematic reviews and meta-analysis (PRISMA) were used (Figure 1). We selected articles of the last 20 years, from 1998 to December 2017. We created an Excel database for collecting data extracted from articles in English language, selecting papers with series of 10 or more patients. Exclusion criteria were articles written in other languages, case reports or reviews, cadaveric or instrumentals studies, clinical studies with no standard questionnaires or scores, and studies in which posttraumatic outcomes were not separated from primary reconstruction of the radial head.

We extracted relevant data from the selected articles: type of study, number of patients, age, follow-up, type of surgery performed, clinical results (ROM, DASH score, MEPS score, and VAS), and radiographic results.

\section{Results}

The database search identified 152 potentially relevant articles. Abstracts have been analyzed following inclusion and exclusion criteria and a total of 29 papers were selected for the present review. Most of retrospective studies on metal radial head prosthesis have been published in the last ten years in comparison to a lack of studies for radial head excision in the last two decades. Moreover, few articles on comparison of the two surgical techniques have been found. Because of heterogeneity in level of evidence, surgical technique, type of implants, and rehabilitation protocol, we did not perform statistical data analysis. Articles selected are reported in Table 1 .

\section{Discussion}

From our review of literature clinical results for radial head replacement are reported in Table 2. Most of retrospective studies involve modular monopolar or bipolar prosthesis implanted for irreparable Mason type III or type IV fractures. For most of authors, mid term follow-up has shown satisfactory results in range of motion recovery (average 
TABLE 1: Studies selected. Number and age of patients, type of treatment, and follow-up.

\begin{tabular}{|c|c|c|c|c|c|}
\hline Author & $\begin{array}{l}\text { Type of study and } \\
\text { year of publication }\end{array}$ & N. of patients & Age & Type of treatment & Follow up \\
\hline Carità E & Retrospective 2017 & $\begin{array}{c}28 \\
\text { (Mason type III - } \\
\text { IV) }\end{array}$ & $\begin{array}{l}49 \text { yo } \\
(18-71)\end{array}$ & $\begin{array}{l}\text { Cementless monopolar prostheses } \\
\text { (Acumed - Tornier) }\end{array}$ & $\begin{array}{l}49 \text { months } \\
(6-118)\end{array}$ \\
\hline Laflamme M & Retrospective 2017 & $\begin{array}{c}46 \\
\text { (21 Mason III; } \\
\text { 36 Mason IV) }\end{array}$ & $\begin{array}{l}\text { Porous stem: } \\
52.8 \text { yo } \\
\text { Smooth stem: } \\
45.6\end{array}$ & $\begin{array}{l}\text { Modular monopolar head - } \\
\text { uncemented loose fitting stem } \\
\text { (Evolve, Wright) } \\
\text { Modular monopolar head - porous } \\
\text { press-fit stem (ExploR, Biomet) }\end{array}$ & $\begin{array}{l}6,3 \text { years } \\
(1,2-15,1)\end{array}$ \\
\hline Tarallo L & Retrospective 2017 & 31 Mason III & - & $\begin{array}{l}\text { 31Radial head replacement } \\
\text { (Anatomic RHA, Acumed) }\end{array}$ & $\begin{array}{c}30 \text { months (12 months } \\
\text { to } 7 \text { years) }\end{array}$ \\
\hline Nestorson J & Restrospective 2017 & 32 Mason IV & $\begin{array}{c}50 \text { yo } \\
(29-70)\end{array}$ & $\begin{array}{l}18 \text { pts radial head arthroplasty } \\
14 \text { radial head resection }\end{array}$ & $\begin{array}{l}58 \text { months (RHA) } \\
108 \text { months (RH } \\
\text { resection) }\end{array}$ \\
\hline $\begin{array}{l}\text { Laumonerie } \\
\mathrm{P}\end{array}$ & Retrospective 2017 & $\begin{array}{l}77 \\
\text { (65 Mason type III, } \\
2 \text { Mason type II; } \\
10 \text { radial neck } \\
\text { fractures) }\end{array}$ & $\begin{array}{c}52 \text { yo } \\
(20-82)\end{array}$ & $\begin{array}{c}\text { Guepar radial head prosthesis } \\
\text { (SBi/Stryker) } \\
\text { Evolutive (Aston Medical) } \\
\text { rHead Recon (SBi/Stryker) } \\
\text { rHead STANDARD (SBi/Stryker) }\end{array}$ & $\begin{array}{l}74 \text { months } \\
(24 \text { to } 141)\end{array}$ \\
\hline Lopiz & Retrospective 2016 & 25 Mason III & $\begin{array}{c}\text { Excixion } \\
53 \text { yo } \\
\text { Arthroplasty } \\
54.4 \text { yo } \\
\end{array}$ & $\begin{array}{l}11 \text { patients radial head resection } \\
14 \text { Radial head prosthesis }\end{array}$ & $\begin{array}{l}\text { Excision } \\
60.3 \text { months } \\
\text { Arthroplasty } \\
42 \text { months }\end{array}$ \\
\hline Van Hoecke & Retrospective 2016 & 21 Mason III & 53,2 yo & Judet bipolar head prosthesis & 113 months \\
\hline Heijink & Retrospective 2016 & 25 Mason type III & 55 уо & $\begin{array}{c}\text { Cemented bipolar radial head } \\
\text { artrhoplasty (Tornier) }\end{array}$ & 50 months \\
\hline Kodde & Retrospective 2016 & 27 & $\begin{array}{c}48 \text { yrs } \\
(24-63)\end{array}$ & $\begin{array}{l}\text { Press fit bipolar radial head } \\
\text { arthroplasty }\end{array}$ & 48 months (28-73) \\
\hline Marsh JP & Retrospective 2016 & 55 & $61 \mathrm{yrs}$ & $\begin{array}{c}\text { Modular smooth-stemmed radial } \\
\text { head implant } \\
\text { (Evolve, Wright) }\end{array}$ & $8.2 \mathrm{yrs}$ \\
\hline Gauci MO & Retrospective 2016 & $\begin{array}{c}65 \\
\text { (10 ORIF revision } \\
42 \text { Mason III } \\
12 \text { post traumatic } \\
\text { radiohumareal } \\
\text { sequelae, } \\
1 \text { swanson } \\
\text { prosthesis revision) }\end{array}$ & $\begin{array}{l}52 \text { yrs } \\
(22-85)\end{array}$ & $\begin{array}{l}\text { Modular Pyrocarbon (MoPyc) } \\
\text { radial head prosthesis (Tornier) }\end{array}$ & 42 months (24-108) \\
\hline Solarino G. & Retrospective 2015 & $\begin{array}{c}30 \\
\text { (12 Mason II; } \\
18 \text { Mason III) } \\
\end{array}$ & 71 yo $(65-80)$ & Radial head resection & 40 months (24-72) \\
\hline Allavena C & Retrospective 2014 & $\begin{array}{c}22 \\
\text { (16 fractures } \\
\text { Mason type III; } \\
6 \text { fractures of the } \\
\text { radial neck) } \\
\end{array}$ & $\begin{array}{l}44 \text { yrs } \\
(22-65)\end{array}$ & $\begin{array}{l}\text { Modular bipolar radial head } \\
\text { prostehesis (Guepar,De Puy) }\end{array}$ & 50 months \\
\hline Yalcinkaya M & Retrospective 2013 & $\begin{array}{c}14 \text { fractures Mason } \\
\text { type III }\end{array}$ & $\begin{array}{l}38 \text { yrs } \\
(20-67) \\
\end{array}$ & Radial head resection & $\begin{array}{c}14,7 \text { yrs } \\
(9-26) \\
\end{array}$ \\
\hline Flinkkila T. & Retrospective 2012 & $\begin{array}{c}42 \\
\text { (34 Mason type III; } \\
8 \text { type II) }\end{array}$ & $\begin{array}{l}56 \text { yrs } \\
(23-85)\end{array}$ & Metallic radial head artrhoplasty & 50 months (12-107) \\
\hline Sarris IK & Retrospective 2012 & $\begin{array}{l}5 \text { Mason type III; } \\
15 \text { type IV; } \\
10 \text { complex elbow } \\
\text { injuries; } \\
2 \text { malunion } \\
\end{array}$ & 54 yrs $(32-68)$ & $\begin{array}{l}\text { MoPyc pyrocarbon prosthesis } \\
\text { (Bioprofile, Tornier) }\end{array}$ & 27 months (21-46) \\
\hline
\end{tabular}


TABLE 1: Continued.

\begin{tabular}{|c|c|c|c|c|c|}
\hline Author & $\begin{array}{l}\text { Type of study and } \\
\text { year of publication }\end{array}$ & N. of patients & Age & Type of treatment & Follow up \\
\hline Ricon F & Retrospective 2012 & 28 Mason III & 54 yrs & $\begin{array}{l}\text { Pyrocarbon radial head prosthesis } \\
\text { (Bioprofile Lab.) }\end{array}$ & 32 months (12-62) \\
\hline Muhm M & Retrospective 2011 & $\begin{array}{l}25 \text { radial head } \\
\text { fractures type III } \\
\text { and type IV }\end{array}$ & - & $\begin{array}{c}\text { Uncemented modular metallic } \\
\text { prosthesis } \\
\text { (Evolve) }\end{array}$ & $\begin{array}{c}\text { Short term } 1,6 y r s \\
\text { Mid term } \\
5,1 \text { yrs } \\
\end{array}$ \\
\hline Iftimie & Retrospective 2011 & $\begin{array}{c}22 \\
\text { (16 Mason type III; } \\
6 \text { type IV) }\end{array}$ & $\begin{array}{l}54 \text { yrs } \\
(28-81)\end{array}$ & Resection head arthroplasty & 16,9 yrs $(10-24)$ \\
\hline Celli A & Retrospective 2010 & $\begin{array}{c}16 \text { patients } \\
\text { (9Mason type III } \\
7 \text { Mason type IV) } \\
\end{array}$ & $\begin{array}{l}46.1 \mathrm{yrs} \\
(27-74)\end{array}$ & $\begin{array}{c}\text { Bipolar Judet radial head } \\
\text { arthroplasty } \\
\text { (Tornier) } \\
\end{array}$ & $\begin{array}{c}41,7 \text { months }(12,3- \\
86,3)\end{array}$ \\
\hline Antuna SA & Retrospective 2010 & $\begin{array}{c}26 \text { patients } \\
(6 \text { type III } \\
20 \text { type IV) }\end{array}$ & $\begin{array}{l}29 \text { yrs } \\
(15-39)\end{array}$ & Radial Head Resection & 24,9 yrs $(15-34)$ \\
\hline Dotzis A & Retrospective 2006 & $\begin{array}{c}14 \text { patients } \\
\text { (6 Mason type III; } \\
8 \text { type IV) }\end{array}$ & $\begin{array}{l}44.8 \text { years } \\
(18-85)\end{array}$ & $\begin{array}{l}\text { Judet prosthesis } \\
\text { (Tornier) }\end{array}$ & $\begin{array}{l}5.3 \text { years } \\
(1-12 \text { yrs })\end{array}$ \\
\hline Ashwood N & Retrospective 2004 & 16 Mason type III & $\begin{array}{l}45 \text { yrs } \\
(21-72)\end{array}$ & $\begin{array}{c}\text { Metallic monoblock radial head } \\
\text { prosthesis } \\
\text { (Wright Med Tec.) }\end{array}$ & $\begin{array}{l}2.8 \text { years } \\
(1.2-4.3)\end{array}$ \\
\hline $\begin{array}{l}\text { Herbertsson } \\
\text { P. }\end{array}$ & Retrospective 2004 & $\begin{array}{l}61 \text { patients } \\
39 \text { Mason type II } \\
10 \text { Mason III } \\
12 \text { Mason IV }\end{array}$ & $\begin{array}{l}44 \text { yrs } \\
(9-69)\end{array}$ & $\begin{array}{c}\text { Radial head resection } \\
\text { Primary } \mathrm{RHE}=39 \\
\text { Delayed } \mathrm{RHE}=18\end{array}$ & $\begin{array}{l}18 \text { years } \\
(11-33)\end{array}$ \\
\hline Moro JK & Retrospective 2001 & $\begin{array}{c}25 \\
\text { (10 Mason type } \\
\text { III;15 Mason type } \\
\text { IV) } \\
\end{array}$ & $54 \mathrm{yrs}$ & Metal Radial head arthroplasty & 39 months \\
\hline $\begin{array}{l}\text { Sanchez } \\
\text { Sotelo J. }\end{array}$ & Retrospective 2000 & 10 Mason type III & $\begin{array}{l}39 \text { yrs } \\
(26-57)\end{array}$ & Radial head resection & $\begin{array}{c}4.62 \text { years }(24-86 \\
\text { months })\end{array}$ \\
\hline Ikeda M & Retrospective 2000 & 11 Mason type III & 40 yrs $(25-70)$ & Radial head resection & $\begin{array}{c}11 \text { years } \\
(3-18) \\
\end{array}$ \\
\hline Smets A & Retrospective 2000 & 13 Mason type III & - & Floating radial head prosthesis & 25.2 months \\
\hline Jansen RP & Retrospective 1998 & 18 Mason III & - & Radial head resection & 16 to 30 years \\
\hline
\end{tabular}

flexion-extension arc of motion: $116^{\circ}$ ). Good results in DASH scores (from 7 to 24) and MEPS scores (from 79 to 100) and low VAS pain evaluation scale (from 0 to 2.2) are reported [13-32]. A certain loss of grip strength compared to contralateral side is often described (average loss of strength: $10 \%$ respect to the contralateral side). Authors highlight the importance of ligament reconstruction in case of associated injuries. Intraoperative assessment of stability and acute repair of torn ligaments is mandatory for a successful procedure.

Most common radiological modifications include osteoarthritic changes of ulnohumeral joint, capitellum wear for oversizing of radial head prosthesis, periarticular heterotopic ossifications, and radiolucency lines around the stem. Some modifications in radiographic appearance seem to not correlate directly with clinical symptoms: bone resorption around the prosthesis does not correlate with loosening of the prosthesis and does not affect clinical scores. Marsh [21] reports favorable clinical outcomes from short to long follow-up despite a high evidence of radiolucency around the stem and arthritis in his series. Gauci [20] has found no association between neck bone resorption and postoperative symptoms.

Complications (Table 3 ) described in radial head replacement are in common in almost all the papers: aseptic mobilization of the stem, overstuffing, erosion of the capitellum, osteoarthritis, and heterotopic ossification clinically arising with lateral elbow pain or loss of motion, and posterior subluxation for undersizing. Rare temporary ulnar and radial nerve sensory neuropathies are reported. Though, few papers seem to discourage radial head arthroplasty. Moro [31] reports mild to moderate impairment of ROM and pain for both elbow and wrist in patients treated with a metal radial head implant. Laumonerie [16] describes unsatisfactory result from bipolar radial head prosthesis because of malposition in varus and valgus and oversizing leading to a high rate of reintervention during the three first months after implantation. Flinkkila [23] reports poor results from press 


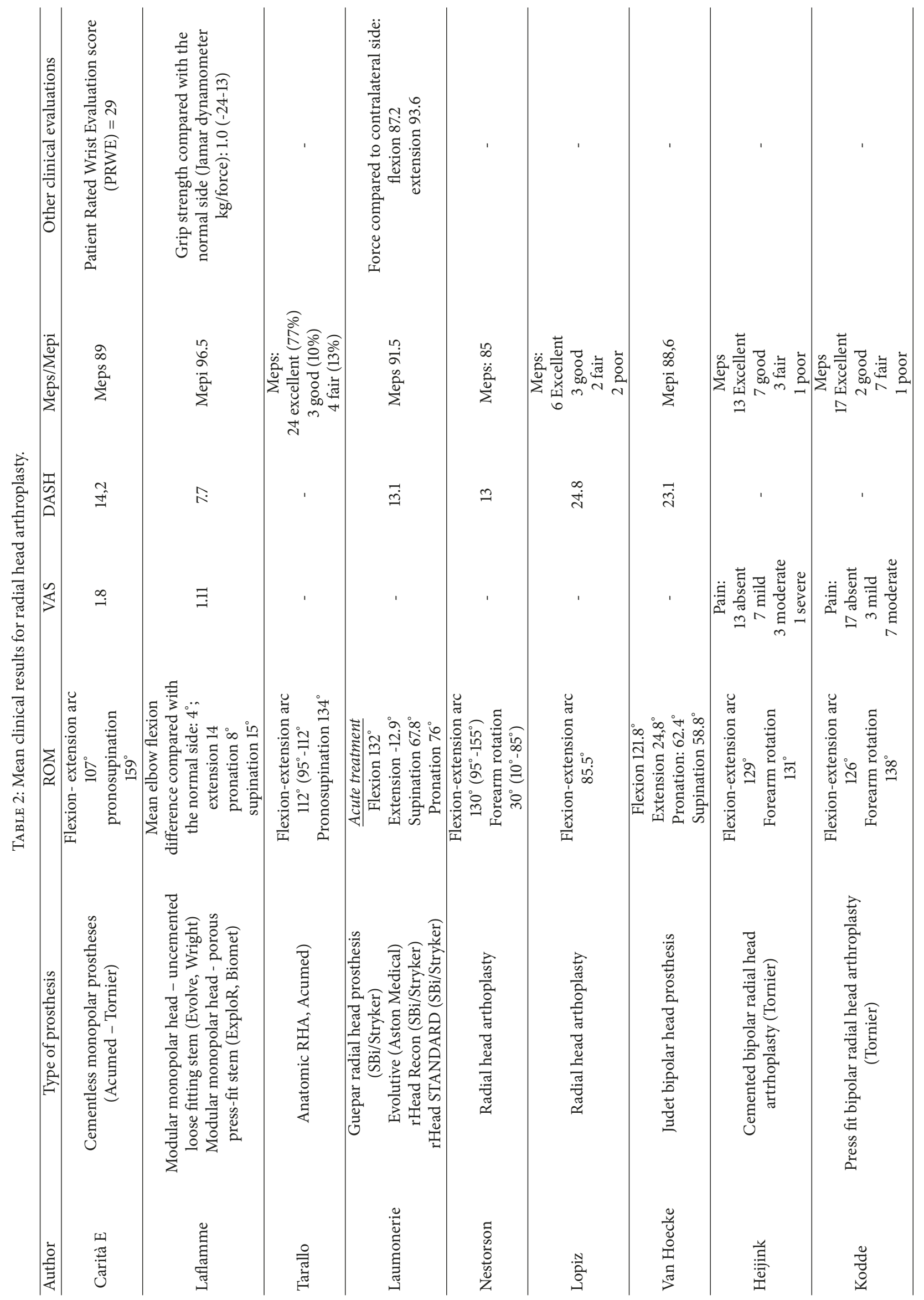




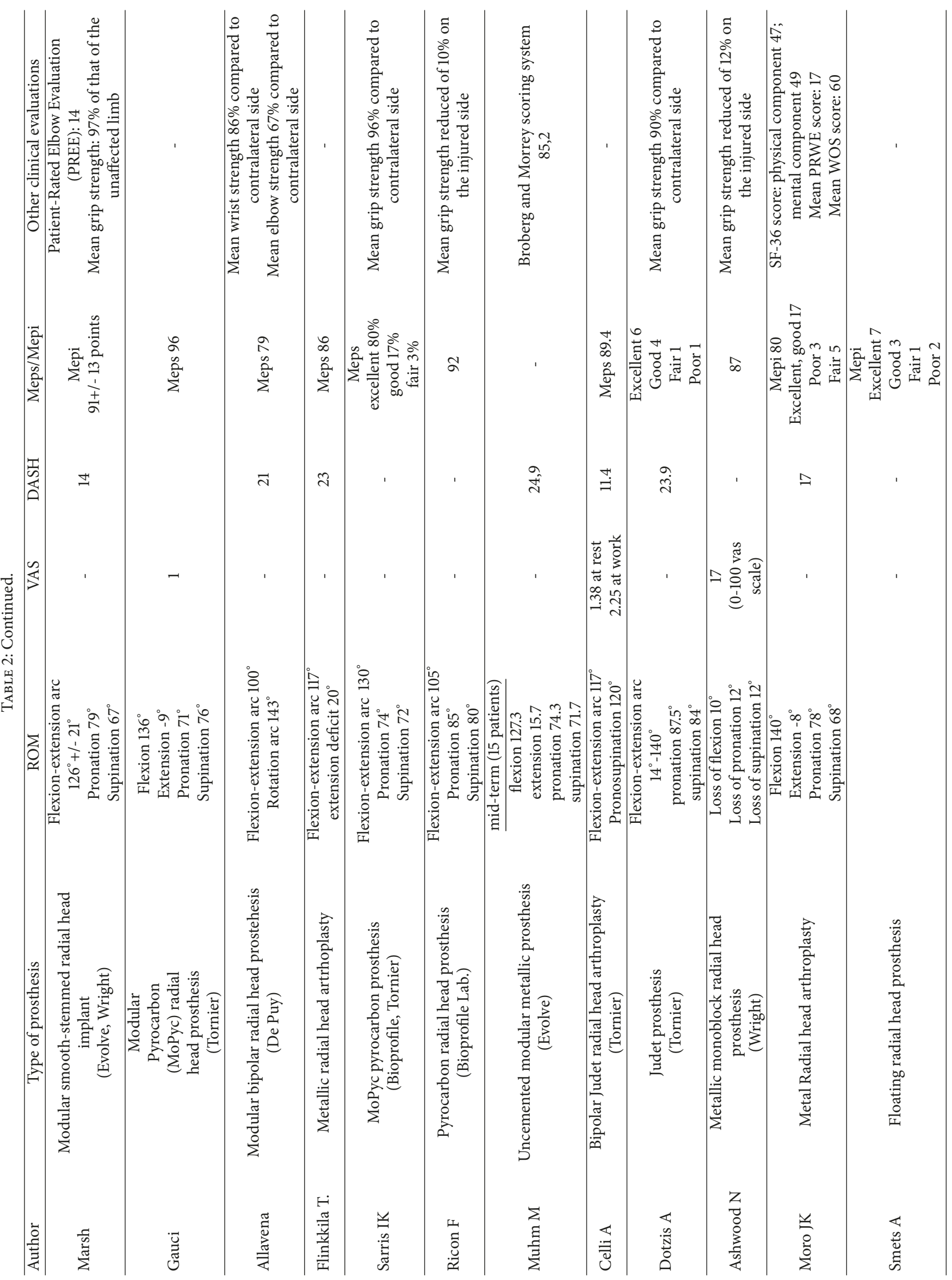


TABLE 3: Complications in radial head replacement.

\begin{tabular}{|c|c|c|}
\hline Author & N. of patients & Complications \\
\hline Carità E & 28 & $\begin{array}{c}1 \text { osteolysis and stem mobilization } \\
1 \text { overstuffing (erosion of the capitellum) } \\
2 \text { periprosthetic calcification(asymptomatic) } \\
6 \text { resorption of the neck of the radius (asymptomatic) } \\
3 \text { removal of the implant (1 mobilization; } 3 \text { painful elbow) }\end{array}$ \\
\hline Laflamme M & 46 & $\begin{array}{c}22 \text { osteolysis }>2 \mathrm{~mm}(48 \%) \\
4 \text { Overstuffing } \\
1 \text { degenerative changes (Broberg and Morrey grade III) } \\
5 \text { heterotopic ossification Brooker grade II, } 1 \text { grade IV }\end{array}$ \\
\hline Tarallo L & 31 & $\begin{array}{c}8 \text { heterotopic ossification }(26 \%) \\
2 \text { radiolucent lines (asymptomatic) }\end{array}$ \\
\hline Nestorson J & 18 & $\begin{array}{c}4 \text { surgical revision ( } 3 \text { aseptic loosening, } 1 \text { proximal } \\
\text { radio-ulnar synostosis, } 1 \text { CPRS) } \\
5 \text { late osteoarthritis }\end{array}$ \\
\hline Laumonerie $\mathrm{P}$ & $\begin{array}{l}54 \\
\text { acute injuries } \\
23 \\
\text { delayed surgery }\end{array}$ & $\begin{array}{c}8 \text { painful loosening } \\
4 \text { radiohumeral conflict } \\
3 \text { radiocapitellar instability } \\
5 \text { ulnar nerve palsy } \\
4 \text { CPRS } \\
30 \text { reoperations (38.9\%) } \\
\text { (19 implants removed; } 11 \text { retention of the implant) }\end{array}$ \\
\hline Lopiz & 14 & $\begin{array}{c}3 \text { elbow stiffness } \\
2 \text { oversizing } \\
1 \text { periprosthetic fracture } \\
2 \text { neuropathies (ulnar and radial) } \\
4 \text { elbow arthritis grade I, } 9 \text { cases grade II, } 1 \text { case grade III } \\
\text { (Broberg and Morrey classification) } \\
11 \text { periarticular ossification (asymptomatic) } \\
5 \text { bone lucencies (asymptomatic) }\end{array}$ \\
\hline Van Hoecke & 21 & $\begin{array}{c}14 \text { capitellar erosion } \\
10 \text { ulnohumeral arthritis } \\
1 \text { radiolucent lines } \\
1 \text { overlenghtening } \\
1 \text { ulnar plus } \\
1 \text { prosthesis removed }\end{array}$ \\
\hline
\end{tabular}

3 radiolucency lines (asymptomatic)

5 periarticular ossification (asymptomatic)

7 osteolysis of proximal radius (asymptomatic)

Heijink

25

4 erosion of the capitellum

13 ulnohumeral arthritis

2 radial nerve neuropraxia

1 luxation (dissociation of the prostheses) - removed 2 subluxation

3 revisions for chronic instability

5 revision for ulnar nerve dysfunction, elbow stiffness, symptomatic arthritis

23 radial neck osteolysis

Kodde IF

13 ulnohumeral degeneration

7 erosion of the capitellum

5 heterotopic ossification (asymptomatic)

1 posterior subluxation

2 persistent pain for medial and lateral epicondylitis

25 periprosthetic lucency

21 ulnohumeral arthritis

Marsh JP

20 heterotopic ossification

12 capitellar osteopenia

1 abnormal radiocapitellar alignment 
TABLE 3: Continued.

\begin{tabular}{|c|c|c|}
\hline Author & N. of patients & Complications \\
\hline Allavena C & 22 & $\begin{array}{c}6 \text { early posterior subluxation } \\
5 \text { sensory ulnar nerve dysfunction } \\
2 \text { CPRS type I } \\
3 \text { lateral elbow pain } \\
1 \text { symptomatic loosening } \\
8 \text { osteolysis } \\
1 \text { advanced osteoarthritis } \\
6 \text { capitellar erosions } \\
4 \text { anterior ossifications }\end{array}$ \\
\hline Flinkkila T. & 42 & $\begin{array}{c}1 \text { infection } \\
1 \text { radial nerve palsy } \\
21 \text { osteoarthritis ( } 3 \text { severe) } \\
14 \text { capitellar erosion } \\
9 \text { prostheses removed (6 painful, } 2 \text { loosed) }\end{array}$ \\
\hline Sarris IK & 32 & $\begin{array}{c}\text { 2 stem-neck dissociation } \\
1 \text { stiffness } \\
2 \text { periprosthetic lucencies (asymptomatic) } \\
7 \text { heterotopic ossification (asymptomatic) } \\
4 \text { radiographic sign of stress shielding (asymptomatic) }\end{array}$ \\
\hline Ricon F & 28 & $\begin{array}{l}2 \text { posterior subluxation (overstuffing) } \\
11 \text { radial neck resorption } \\
5 \text { ossification in collateral ligament } \\
1 \text { mild periprosthetic ossification }\end{array}$ \\
\hline Muhm M & $\frac{\text { Mid term }}{15}$ & $\begin{array}{c}12 \text { periprosthetic radiolucency } \\
12(70 \%) \text { heterotopic ossification } \\
12(70 \%) \text { osteoarthritis }\end{array}$ \\
\hline Iftimie & 22 & 24 degenerative changes \\
\hline Celli A & 16 & $\begin{array}{c}2 \text { heterotopic ossification } \\
2 \text { proximal radio-ulnar synostosis } \\
2 \text { capitellar erosion (overstuffing) } \\
1 \text { proximal bone resorption }\end{array}$ \\
\hline Dotzis A & 14 & $\begin{array}{c}1 \text { CPRS and stiffness } \\
1 \text { periprosthetic lucency } \\
7 \text { heterotopic ossification (asymptomatic) }\end{array}$ \\
\hline Ashwood N & 16 & $\begin{array}{c}1 \text { CPRS } \\
3 \text { ulnar neuropathies } \\
2 \text { superficial wound infections }\end{array}$ \\
\hline Moro JK & 25 & $\begin{array}{c}17 \text { bone radiolucency (asymptomatic) } \\
1 \text { CPRS } \\
1 \text { ulnar neuropathy } \\
1 \text { PIN palsy } \\
1 \text { elbow stiffness } \\
1 \text { wound infection }\end{array}$ \\
\hline Smets A & 13 & $\begin{array}{c}3 \text { wrist pain } \\
1 \text { implant removed for stiffness }\end{array}$ \\
\hline
\end{tabular}

fit radial head prosthesis due to a high rate of loosening. Difficulties on technique of implantation are described by Ashwood [30] for mono-block prosthesis.

Retrospective studies on radial head resection have a longer follow-up and clinical and radiological results are reported in Table 4 [33-42]. Clinical and radiological complications at long-term follow-up are reported (Table 5). Clinical results show good outcomes in Mayo Elbow Performance Scores (MEPS, from 79 to 100) and Disabilities for Arm Shoulder and Harm scores (DASH, from 4 to 15), a satisfactory recovery of elbow range of motion (average flexion-extension arc of motion: $120^{\circ}$ ) and low scores in VAS scale (from 0 to 4.6). However common complications of this surgical procedure involve ulnohumeral joint due to an higher load compression force that leads to degenerative changes and progressive worsening of cubitus valgus associated to ulnar nerve neuropathy and UCL elongation leading to chronic elbow instability [3, 4]. Moreover, proximal migration of radius is often assessed $(80 \%$ of papers), complications that involve DRUJ impairment 
TABLE 4: Mean clinical results for radial head resection.

\begin{tabular}{|c|c|c|c|c|c|}
\hline Author & $\mathrm{ROM}$ & VAS & DASH & Meps/Mepi & Other clinical evaluations \\
\hline Nestorson J & $\begin{array}{c}\text { Flexion-extension arc } \\
127,5^{\circ}\left(105^{\circ}-150^{\circ}\right)\end{array}$ & - & 12 & Meps: 100 & - \\
\hline Lopiz & $\begin{array}{l}\text { Flexion-extension arc } \\
105.2^{\circ}\end{array}$ & - & 13.5 & $\begin{array}{c}\text { Meps } \\
6 \text { excellent } \\
3 \text { good } \\
2 \text { fair } \\
0 \text { poor }\end{array}$ & - \\
\hline Solarino G. & $\begin{array}{l}\text { flexion } 124^{\circ} \\
\text { extension }-11^{\circ} \\
\text { pronation } 78^{\circ} \\
\text { supination } 82^{\circ}\end{array}$ & $\begin{array}{l}\text { Pain Absent } 14 \\
\text { Mild } 8 \\
\text { Moderate } 8\end{array}$ & 13 & Meps 79 & - \\
\hline Yalcinkaya M & $\begin{array}{l}\text { Flexion-extension arc } \\
\qquad 127^{\circ} \\
\text { Pronation } 83,2^{\circ} \\
\text { Supination } 84,6^{\circ} \\
\end{array}$ & 4,6 & 6,6 & Meps 88,6 & - \\
\hline Iftimie & $\begin{array}{l}\text { flexion } 135^{\circ} \\
\text { extension }-5^{\circ} \\
\text { pronation } 83^{\circ} \\
\text { supination } 79^{\circ} \\
\end{array}$ & 0.48 & 4,89 & - & $\begin{array}{l}\text { Grip strength } 88 \% \text { compared to the } \\
\text { contralateral side }\end{array}$ \\
\hline Antuna SA & $\begin{array}{l}\text { flexion } 84^{\circ} \\
\text { extension }-9^{\circ} \\
\text { pronation } 84^{\circ} \\
\text { supination } 85^{\circ} \\
\end{array}$ & 9 & 6 & 95 & $\begin{array}{l}\text { Grip strength loss } 16 \% \text { compared to } \\
\text { contralateral side }\end{array}$ \\
\hline $\begin{array}{l}\text { Herbertsson } \\
\text { P. }\end{array}$ & $\begin{array}{l}\text { Primary } R H A \\
\text { flexion } 140^{\circ} \\
\text { extension }-7^{\circ} \\
\text { supination } 77^{\circ} \\
\text { pronation } 85^{\circ}\end{array}$ & - & - & - & $\begin{array}{l}\text { Steinberg system for clinical outcomes: } \\
25 \text { good; } 26 \text { fair; } 10 \text { poor } \\
28 \text { no symptoms, } 27 \text { occasional pain; } 6 \\
\text { daily pain }\end{array}$ \\
\hline $\begin{array}{l}\text { Sanchez } \\
\text { Sotelo J. }\end{array}$ & $\begin{array}{l}\text { Flexion-extension } 7.5- \\
\quad 140 \\
\text { Pronation } 85.5^{\circ} \\
\text { Supination } 83.5^{\circ}\end{array}$ & 0 & 0.66 to 15.9 & - & $\begin{array}{l}\text { Grip strength loss } 15 \% \text { compared to } \\
\text { contralateral side } \\
\text { Broberg and Morrey performance index: } \\
\text { excellent 5; good 5; poor } 1\end{array}$ \\
\hline Ikeda M & $\begin{array}{l}\text { flexion } 132^{\circ} \\
\text { extension }-14^{\circ} \\
\text { supination } 82^{\circ} \\
\text { pronation } 80^{\circ}\end{array}$ & - & - & - & $\begin{array}{l}\text { Grip strength loss } 17 \% \text { compared to } \\
\text { contralateral side }\end{array}$ \\
\hline Jansen RP & - & - & - & $\begin{array}{c}\text { Mepi } \\
\text { Excellent } 17 \\
\text { Good } 3 \\
\text { Fair } 1 \\
\text { Poor } 2\end{array}$ & - \\
\hline
\end{tabular}

leading to wrist pain hand strength reduction and distal radio-ulnar arthritis. Preoperative or intraoperative setting of elbow stability and correction of ligaments injuries is mandatory to avoid early complications. Despite of complications, many authors approve the surgical technique due to good outcomes in mid to large term. Yalcinkaya [36] found no significant correlation between radiological degenerative modifications in elbow and outcomes of clinical scores in patients treated by radial head resection. Antuna [38] reports good clinical results in a large series of patients less than forty years old treated by radial head excision after a mean follow-up of 25 years. Herbertsson [39] reports worst outcomes in excision for Mason type IV fractures although delayed radial arthroplasty is suggested for pain relief and preservation of range of motion in case of failure of other treatments.

Finally, few papers compare radial head resection and radial head arthroplasty $[34,35]$ where authors recommend resection as primary option of treatment because of a lack of statistical clinical differences between the two surgical procedures, in case of isolated radial head fractures not associated to ligaments injuries. Nestorson [33] did not found better outcomes by using a press fit radial head prosthesis in Mason type IV fractures and he reports similar functional results after radial head resection despite more osteoarthritic changes. Lopiz [34] suggests resection as a good option of 
TABLE 5: Complications in radial head resection.

\begin{tabular}{|c|c|c|}
\hline Author & N. Of patients & Complications \\
\hline Nestorson J & 14 & $\begin{array}{c}2 \text { surgical revision (stiffness) } \\
1 \text { ulnar nerve dysfunction } \\
1 \text { radial nerve dysfunction } \\
13 \text { late osteoarthritis }\end{array}$ \\
\hline Lopiz & 11 & $\begin{array}{c}\text { Average radial shortening } 2.3 \mathrm{~mm} \\
1 \text { elbow stiffness } \\
1 \text { valgus instability } \\
\text { All patients: elbow arthritis grade I } \\
2 \text { heterotopic ossification (asymptomatic) }\end{array}$ \\
\hline Solarino G. & 30 & $\begin{array}{c}\text { Arthritic changes: } 4 \text { mild; } 3 \text { moderate } \\
5 \text { heterotopic ossification } \\
5 \text { ulnar minus (mean value } 3.5 \text { ) and DRUJ } \\
\text { instability }\end{array}$ \\
\hline Yalcinkaya M & 14 & $\begin{array}{c}8 \text { degenerative changes in elbow } \\
4 \text { degenerative changes in wrist } \\
3 \text { heterotopic ossification } \\
8 \text { proximal migration of radius } \\
\text { Mean ulnar variance: } 1.7 \mathrm{~mm} \\
\text { Mean carrying angle } 11.2^{\circ} \\
\end{array}$ \\
\hline Iftimie & 22 & $\begin{array}{c}24 \text { Degenerative changes (Broberg and Morrey } \\
1 \text { patient grade } 3 ; 13 \text { grade } 2 ; 10 \text { grade } 1\end{array}$ \\
\hline Antuna SA & 26 & $\begin{array}{c}2 \text { postero-lateral instability } \\
2 \text { valgus laxity } \\
1 \text { DRUJ instability } \\
\text { Osteoarthritic changes (17 mild; } 9 \text { moderate) } \\
8 \text { heterotopic ossification (asymptomatic) } \\
\text { Average radial shortening } 3.1 \mathrm{~mm}\end{array}$ \\
\hline Herbertsson P. & 61 & $\begin{array}{c}16 \text { ulnar plus }>2 \mathrm{~mm} \\
\text { Degenerative changes: } 42 \text { cysts; } 40 \text { irregular } \\
\text { subchondral bone; } 43 \text { osteophytes }\end{array}$ \\
\hline Sanchez Sotelo J. & 10 & $\begin{array}{c}4 \text { heterotopic ossification } \\
8 \text { degenerative arthritis } \\
\text { mean proximal radius migration: } 1.6 \mathrm{~mm} \\
\text { mean carrying angle decrease: } 5.4^{\circ}\end{array}$ \\
\hline Ikeda M & 11 & $\begin{array}{c}\text { Mean ulnar variance }+1.6 \mathrm{~mm} \\
\text { Mean increase in carrying angle } 8^{\circ} \\
\text { Mild to severe degenerative arthritis in all } \\
\text { patients }\end{array}$ \\
\hline Jansen RP & 18 & $\begin{array}{c}\text { ROM limitations } \\
11 \text { Degenerative changes } \\
7 \text { increase of cubitus valgus, } \\
7 \text { periarticular ossification, } \\
7 \text { osteoporosis of capitellum, } \\
12 \text { proximal radius migration (from } 1 \text { to } 5 \mathrm{~mm} \text { ) }\end{array}$ \\
\hline
\end{tabular}

treatment when ORIF is not possible, reporting a higher rate of complications in the group of patient treated by radial head arthroplasty.

\section{Conclusion}

From our review of literature almost all the retrospective studies on radial head arthroplasty report convincing results in terms of elbow stability, range of motion, and pain. Nevertheless, papers on radial head resection report good clinical outcomes in isolated radial head resection with no associated ligament injuries. Few papers compare the two techniques with no substantial differences in terms of clinical outcomes at medium and long follow-up.

\section{Conflicts of Interest}

The authors declare that there are no conflicts of interest regarding the publication of this paper.

\section{References}

[1] B. F. Morrey, K. N. An, and T. J. Stormont, "Force transmission through the radial head." The Journal of Bone \& Joint Surgery, vol. 70, no. 2, pp. 250-256, 1988. 
[2] S. L. Jensen, B. S. Olsen, S. Tyrdal, J. O. Søjbjerg, and O. Sneppen, "Elbow joint laxity after experimental radial head excision and lateral collateral ligament rupture: Efficacy of prosthetic replacement and ligament repair," Journal of Shoulder and Elbow Surgery, vol. 14, no. 1, pp. 78-84, 2005.

[3] R. P. Van Riet, "Associated injuries complicating radial head fractures: a demographic study," Clinical Orthopaedics and Related Research, 2005.

[4] N. F. Hilgersom, D. Eygendaal, and M. P. van den Bekerom, "Is radial head resection the first choice treatment of comminuted radial head fractures without associated instability?" Injury, vol. 48, no. 2, pp. 560-562, 2017.

[5] J. Itamura, N. Roidis, R. Mirzayan, S. Vaishnav, T. Learch, and C. Shean, "Radial head fractures: MRI evaluation of associated injuries," Journal of Shoulder and Elbow Surgery, vol. 14, no. 4, pp. 421-424, 2005.

[6] J. C. McGinley, G. Gold, E. Cheung, and J. Yao, "MRI Detection of Forearm Soft Tissue Injuries with Radial Head Fractures," HAND, vol. 9, no. 1, pp. 87-92, 2014.

[7] B. F. Morrey, S. Tanaka, and K. An, "Valgus Stability of the Elbow," Clinical Orthopaedics and Related Research, no. 265, pp. 187-195, 1991.

[8] N. Bonnevialle, "Radial head replacement in adults with recent fractures," Orthopaedics \& traumatology, surgery \& research : OTSR, vol. 102, no. 1, pp. S69-S79, 2016.

[9] Z. D. Mikic and S. M. Vukadinovic, "Late results in fractures of the radial head treated by excision," Clinical Orthopaedics and Related Research, vol. 181, pp. 220-228, 1983.

[10] G. Giannicola, F. M. Sacchetti, G. Antonietti, A. Piccioli, R. Postacchini, and G. Cinotti, "Radial head, radiocapitellar and total elbow arthroplasties: A review of recent literature," Injury, vol. 45, no. 2, pp. 428-436, 2014.

[11] A. L. Lindenhovious, "Open reduction and internal fixation compared with excision for unstable displaced fractures of the radial head," The Journal of Hand Surgery, 2007.

[12] M. N. Kaur, "Functional outcomes post-radial head artrhoplasty: a systematic review of literature," Shoulder and Elbow, 2014.

[13] E. Carità, A. Donadelli, L. Cugola, and P. Perazzini, "Radial head prosthesis: results overview," Musculoskeletal Surgery, vol. 101, pp. 197-204, 2017.

[14] M. Laflamme, P.-P. Grenier-Gauthier, A. Leclerc, S. Antoniades, and A.-M. Bédard, "Retrospective cohort study on radial head replacements comparing results between smooth and porous stem designs," Journal of Shoulder and Elbow Surgery, vol. 26, no. 8, pp. 1316-1324, 2017.

[15] L. Tarallo, R. Mugnai, M. Rocchi, F. Capra, and F. Catani, "Mason type III radial head fractures treated by anatomic radial head arthroplasty: Is this a safe treatment option?" Orthopaedics \& Traumatology: Surgery \& Research, vol. 103, no. 2, pp. 183-189, 2017.

[16] Laumonerie, "Mid term oucome of 77 modular radial head prostheses," Bone Joint Journal, 2017.

[17] V. Hoecke, "Long term results after bipolar radial head arthroplasty," Acta Orthopaedica Belgica, 2016.

[18] A. Heijink, I. F. Kodde, P. G. H. Mulder, C. N. Van Dijk, and D. Eygendaal, "Cemented bipolar radial head arthroplasty: midterm follow-up results," Journal of Shoulder and Elbow Surgery, vol. 25, no. 11, pp. 1829-1838, 2016.

[19] Kodde, "Press-fit bipolar radial head arthroplasty, mide term results," Journal of Shoulder and Elbow Surgery, 2016.
[20] M.-O. Gauci, M. Winter, C. Dumontier, N. Bronsard, and Y. Allieu, "Clinical and radiologic outcomes of pyrocarbon radial head prosthesis: Midterm results," Journal of Shoulder and Elbow Surgery, vol. 25, no. 1, pp. 98-104, 2016.

[21] J. P. Marsh, R. Grewal, K. J. Faber, D. S. Drosdowech, G. S. Athwal, and G. J. King, "Radial Head Fractures Treated with Modular Metallic Radial Head Replacement," The Journal of Bone \& Joint Surgery, vol. 98, no. 7, pp. 527-535, 2016.

[22] C. Allavena, S. Delclaux, N. Bonnevialle, M. Rongières, P. Bonnevialle, and P. Mansat, "Outcomes of bipolar radial head prosthesis to treat complex radial head fractures in 22 patients with a mean follow-up of 50 months," Orthopaedics \& Traumatology: Surgery \& Research, vol. 100, no. 7, pp. 703-709, 2014.

[23] T. Flinkkilä, T. Kaisto, K. Sirniö, P. Hyvönen, and J. Leppilahti, "Short- to mid-term results of metallic press-fit radial head arthroplasty in unstable injuries of the elbow," The Journal of Bone \& Joint Surgery (British Volume), vol. 94, no. 6, pp. 805810, 2012.

[24] I. K. Sarris, M. J. Kyrkos, N. N. Galanis, K. A. Papavasiliou, F. E. Sayegh, and G. A. Kapetanos, "Radial head replacement with the MoPyC pyrocarbon prosthesis," Journal of Shoulder and Elbow Surgery, vol. 21, no. 9, pp. 1222-1228, 2012.

[25] F. J. Ricón, P. Sánchez, F. Lajara, A. Galán, J. A. Lozano, and E. Guerado, "Result of a pyrocarbon prosthesis after comminuted and unreconstructable radial head fractures," Journal of Shoulder and Elbow Surgery, vol. 21, no. 1, pp. 82-91, 2012.

[26] M. Muhm, R. de Castro, and H. Winkler, "Radial head arthroplasty with an uncemented modular metallic radial head prosthesis: Short- and mid-term results," European Journal of Trauma and Emergency Surgery, vol. 37, no. 1, pp. 85-95, 2011.

[27] A. Celli, F. Modena, and L. Celli, "The acute bipolar radial head replacement for isolated unreconstructable fractures of the radial head," Musculoskeletal Surgery, vol. 94, pp. S3-S9, 2010.

[28] A. Dotzis, G. Cochu, C. Mabit, J. L. Charissoux, and J. P. Arnaud, "Comminuted fractures of the radial head treated by the Judet floating radial head prosthesis," The Journal of Bone \& Joint Surgery (British Volume), vol. 88, no. 6, pp. 760-764, 2006.

[29] J.-M. Brinkman, F. T. G. Rahusen, M. J. De Vos, and D. Eygendaal, "Treatment of sequelae of radial head fractures with a bipolar radial head prosthesis: Good outcome after 1-4 years follow-up in 11 patients," Acta Orthopaedica, vol. 76, no. 6, pp. 867-872, 2005.

[30] N. Ashwood, G. I. Bain, and R. Unni, "Management of Mason Type-III Radial Head Fractures with a Titanium Prosthesis, Ligament Repair, and Early Mobilization," The Journal of Bone \& Joint Surgery, vol. 86, no. 2, pp. 274-280, 2004.

[31] J. K. Moro, J. Werier, J. C. MacDermid, S. D. Patterson, and G. J. W. King, "Arthroplasty with a metal radial head for unreconstructible fractures of the radial head," The Journal of Bone \& Joint Surgery, vol. 83, no. 8, pp. 1201-1211, 2001.

[32] S. Smets, "The floating radial head prosthesis for comminuted radial head fractures: a multicentric study," Acta Orthopaedica Belgica, 2000.

[33] J. Nestorson, P.-O. Josefsson, and L. Adolfsson, "A radial head prosthesis appears to be unnecessary in Mason-IV fracture dislocation," Acta Orthopaedica, vol. 88, no. 3, pp. 315-319, 2017.

[34] Y. Lópiz, A. González, C. García-Fernández, J. García-Coiradas, and F. Marco, "Comminuted fractures of the radial head: resection or prosthesis?” Injury, vol. 47, pp. S29-S34, 2016.

[35] G. Solarino, G. Vicenti, A. Abate, M. Carrozzo, G. Picca, and B. Moretti, "Mason type II and III radial head fracture in patients 
older than 65: is there still a place for radial head resection?" Aging Clinical and Experimental Research, vol. 27, pp. 77-83, 2015.

[36] M. Yalcinkaya, A. E. Bagatur, S. Erdogan, and G. Zorer, "Resection arthroplasty for mason type III radial head fractures yield good clinical but poor radiological results in the long term," Orthopedics, vol. 36, no. 11, pp. e1358-e1364, 2013.

[37] P. P. Iftimie, J. Calmet Garcia, I. de Loyola Garcia Forcada, J. E. Gonzalez Pedrouzo, and J. Giné Gomà, "Resection arthroplasty for radial head fractures: Long-term follow-up," Journal of Shoulder and Elbow Surgery, vol. 20, no. 1, pp. 45-50, 2011.

[38] S. A. Antuña, J. M. Sánchez-Márquez, and R. Barco, "Long-term results of radial head resection following isolated radial head fractures in patients younger than forty years old," The Journal of Bone \& Joint Surgery, vol. 92, no. 3, pp. 558-566, 2010.

[39] P. Herbertsson, P. O. Josefsson, R. Hasserius, J. Besjakov, F. Nyqvist, and M. K. Karlsson, "Fractures of the radial head and neck treated with radial head excision," The Journal of Bone \& Joint Surgery, vol. 86, no. 9, pp. 1925-1930, 2004.

[40] J. Sanchez-Sotelo, O. Romanillos, and E. G. Garay, "Results of acute excision of the radial head in elbow radial head fracturedislocations," Journal of Orthopaedic Trauma, vol. 14, no. 5, pp. 354-358, 2000.

[41] M. Ikeda and Y. Oka, "Function after early radial head resection for fracture: A retrospective evaluation of 15 patients followed for 3-18 years," Acta Orthopaedica, vol. 71, no. 2, pp. 191-194, 2000.

[42] R. P. Janssen and J. Vegter, "Resection of the radial head after Mason type-III fractures of the elbow," The Journal of Bone \& Joint Surgery (British Volume), vol. 80-B, no. 2, pp. 231-233, 1998. 


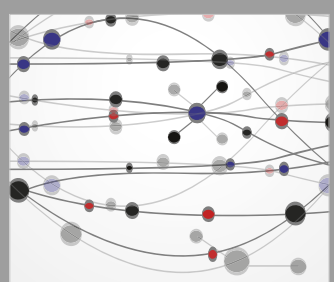

The Scientific World Journal
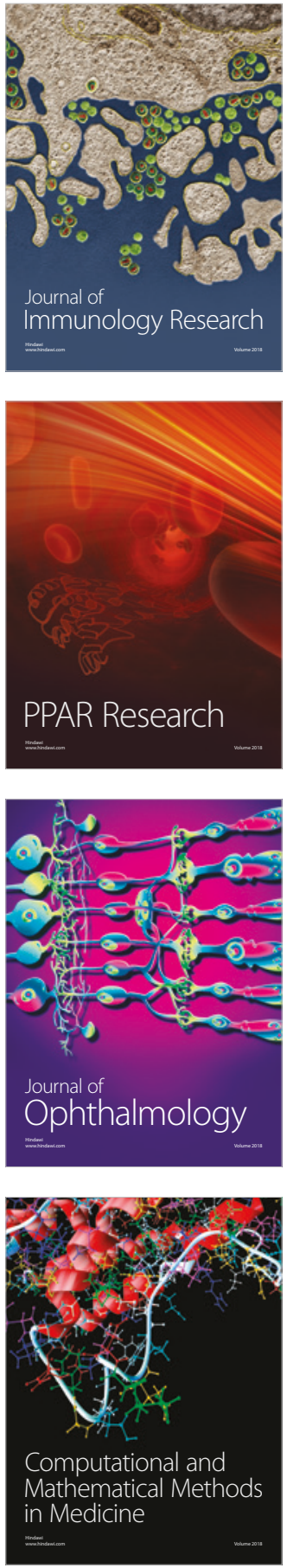

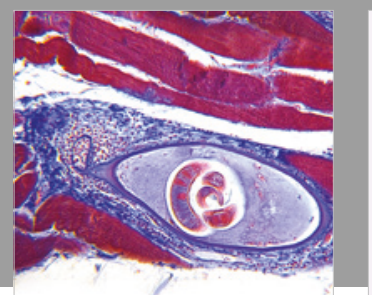

Gastroenterology Research and Practice

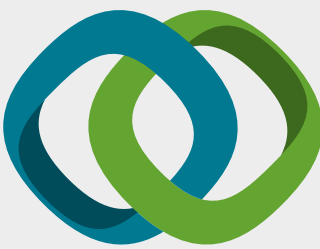

\section{Hindawi}

Submit your manuscripts at

www.hindawi.com
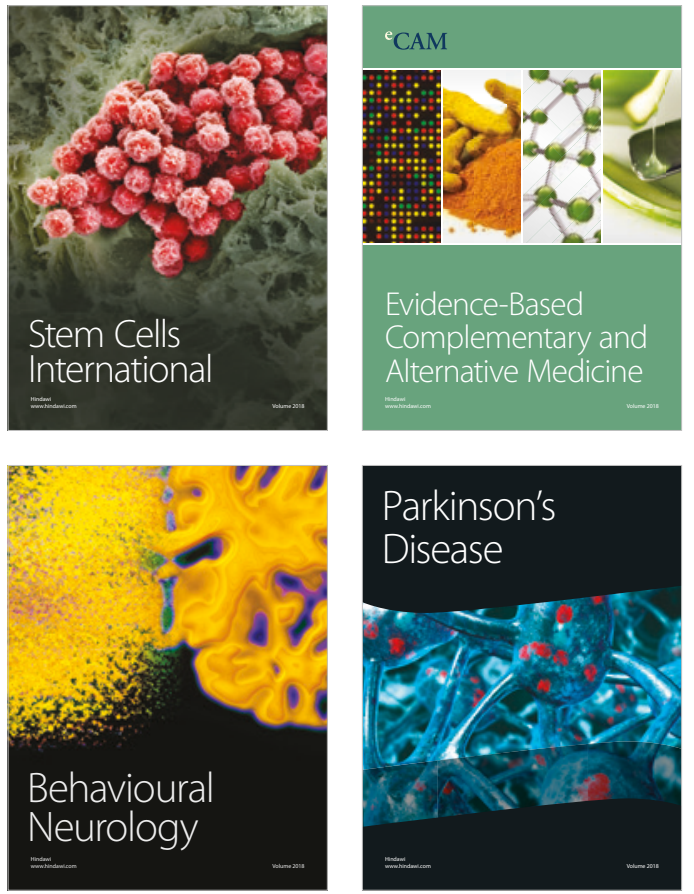

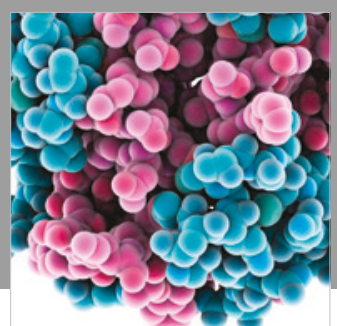

ournal of

Diabetes Research

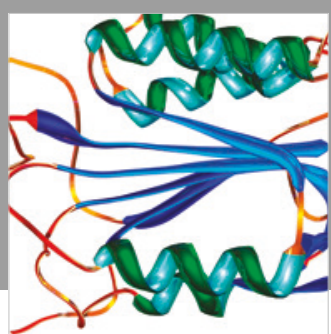

Disease Markers
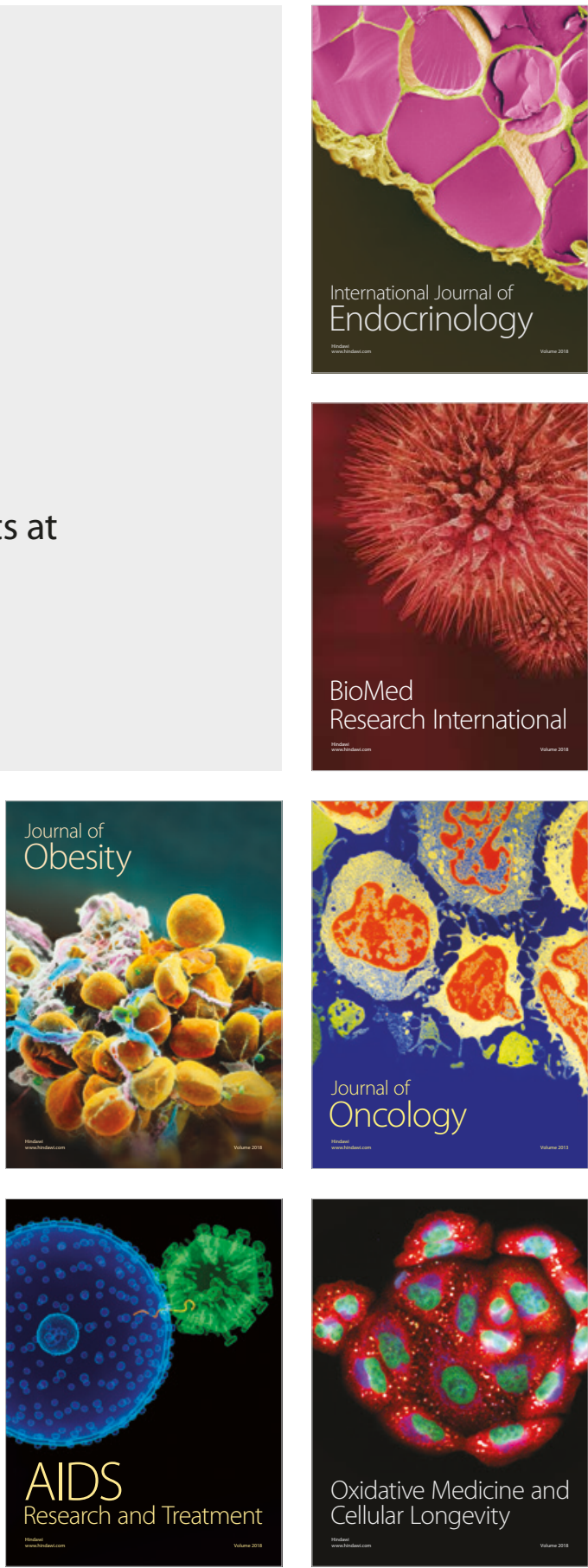